Pacific Journal of Mathematics

OPERATORS OF RIESZ TYPE 


\section{OPERATORS OF RIESZ TYPE}

\section{S. R. CARAduS}

The concept of an operator of Riesz type was introduced by A. F. Ruston by using as an axiomatic system those properties of compact operator used by $F$. Riesz in his original discussion of integral equations. In this paper we first show that this system of axioms can be somewhat simplified, and that in fact the class $\mathscr{R}$ of operators of Riesz type coincides with the class of bounded linear operators whose Fredholm region consists of all nonzero complex numbers. It is further shown that the class of strictly singular operators introduced by $T$. Kato and the class of inessential operators introduced by $D$. C. Kleinecke both lie within $\mathscr{R}$. Next, perturbation theory is considered and it is shown that with suitable commutativity conditions, $\mathscr{R}$ has the defining properties of a closed ideal. Finally, if $f$ is analytic on an open set containing $\sigma(T)$ and $f(0)=0$, then $f(T) \in \mathscr{R}$ if $T \in \mathscr{R}$. Moreover, if $T \in \mathscr{R}$, then the algebra generated by $T$ also lies within $\mathscr{R}$.

Let $X$ denote an arbitrary complex Banach space and $B(X)$ the space of bounded linear operators on $X$. For $T \in B(X)$, define the null manifold $N(T)$ and the range $R(T)$, also the ascent $\alpha(T)$ and the descent $\delta(T)$ as in [13]. We shall write $n(T)$ to denote the nullity of $T$, i.e., $\operatorname{dim} N(T)$, and $d(T)$ for the defect of $T$, i.e., the codimension of $R(T)$. Finally we define $\bar{d}(T)$, the closed defect of $T$, as the codimension of the uniform closure of $R(T)$. We shall not distinguish between infinite cardinals.

The five quantities $\alpha(T), \delta(T), n(T), d(T)$ and $\bar{d}(T)$ are useful in discussions of linear operators. It has been known for many years that if $\alpha(T)$ and $\delta(T)$ are finite, then they are equal. More recently, T. Kato showed [7] that if $d(T)$ is finite, then $d(T)=\bar{d}(T)$. This is clearly equivalent to concluding that $R(T)$ is closed. A more systematic attempt to relate the first four of our quantities is found in the dissertation [5] of $\mathrm{H}$. Heuser. Two important relations discovered were as follows:

(1) Suppose at least one of the quantities $n(T)$ and $d(T)$ is finite. Then

$$
\begin{aligned}
& \text { (a) if } \alpha(T)<\infty \text {, then } d(T) \geqq n(T) \text {; } \\
& \text { (b) if } \delta(T)<\infty \text {, then } d(T) \leqq n(T) \text {. }
\end{aligned}
$$

(2) Suppose $n(T)=d(T)<\infty$ and one of the quantities $\alpha(T)$, $\delta(T)$ is finite. Then the other of these two quantities is finite. 
If we define $\rho(T), P_{\sigma}(T), C_{\sigma}(T)$ and $R_{\sigma}(T)$ as in [13], and we write $\alpha(\lambda), \delta(\lambda)$, etc. for $\alpha(\lambda-T), \delta(\lambda-T)$ etc., then the following table gives a useful characterisation.

\begin{tabular}{c|rrrrr}
$\lambda$ & $\alpha(\lambda)$ & $\delta(\lambda)$ & $n(\lambda)$ & $d(\lambda)$ & $\bar{d}(\lambda)$ \\
\hline$\rho(T)$ & 0 & 0 & 0 & 0 & 0 \\
$P_{\sigma}(T)$ & $\neq 0$ & & $\neq 0$ & & \\
$C_{\sigma}(T)$ & 0 & $\infty$ & 0 & $\infty$ & 0 \\
$R_{\sigma}(T)$ & 0 & $\infty$ & 0 & $\neq 0$ & $\neq 0$
\end{tabular}

To verify this table, we begin with the elementary observation that $\alpha(\lambda)=0$ if and only if $n(\lambda)=0$ and $\delta(\lambda)=0$ if and only if $d(\lambda)=0$. If $\lambda \in \rho(T), \lambda-T$ has an inverse in $B(X)$ and it follows that all entries must be zero. For $\lambda \in P_{\sigma}(T), N(\lambda-T) \neq\{0\}$. Hence $n(\lambda)$ and consequently $\alpha(\lambda)$ are nonzero. If $\lambda \in C_{\sigma}(T), N(\lambda-T)=\{0\}$, $R(\lambda-T) \neq X$ but $\overline{R(\lambda-T)}=X$. We then deduce the entries in the table using the result of Kato cited above. Finally, if $\lambda \in R_{\sigma}(T)$, $N(\lambda-T)=\{0\}, \overline{R(\lambda-T)} \neq X$. Using Heuser's result (2), we conclude this proof.

Riesz Operators. A. F. Ruston [12] defines $T$ to be an operator of Riesz type in $B(X)$ if the following requirements are fulfilled:

$R 1: \quad \alpha(\lambda)$ and $\delta(\lambda)$ are finite for all $\lambda \neq 0$,

R2: $n\left[(\lambda-T)^{\alpha(\lambda)}\right]$ is finite for all $\lambda \neq 0$,

$R 3: \quad R\left[(\lambda-T)^{n}\right]$ is closed for all $\lambda \neq 0$ and all positive integers $n$,

$R 4: P_{\sigma}(T)$ has no nonzero points of accumulation.

We shall denote the class of Riesz operators by $\mathscr{R}$. It is well known that $\mathscr{R}$ contains $\mathscr{C}$, the class of compact operators in $B(X)$.

We begin by showing that $R 3$ and $R 4$ are redundant and that $R 2$ can be replaced by a somewhat simpler requirement.

Lemma 1. (i) $R 1$ and $R 2$ imply $R 3$, (ii) $R 1$ and $R 3$ imply $R 4$.

Proof. (i) Assume $R 1$ and $R 2$ and let $\lambda \neq 0$. Then by $R 1$, $\alpha(\lambda)$ and $\delta(\lambda)$ are finite and hence equal. Call their common value $p$. Let $n$ be any positive integer and write $S=(\lambda-T)^{n}$. Then by $R 2$, $n(S)$ is finite since $N(S) \leqq N\left[(\lambda-T)^{p}\right]$. Moreover, $\alpha(S)=\delta(S) \leqq p$. Hence by (1), $d(S)$ is finite and thus $R(S)$ is closed.

(ii) Assume $R 1$ and $R 3$. Let $\lambda_{0}$ be a nonzero point of accumulation of $P_{\sigma}(T)$. Then there exist eigenvalues $\lambda_{1}, \lambda_{2}, \cdots$ of $T$ such 
that $\lambda_{n} \rightarrow \lambda_{0}$ and $\lambda_{n} \neq \lambda_{0}$ and eigenvectors $x_{1}, x_{2}, \cdots$ in $X$ such that $T x_{n}=\lambda_{n} x_{n}$ and $\left\|x_{n}\right\|=1$. Now $\left(\lambda_{0}-T\right) x_{n}=\left(\lambda_{0}-\lambda_{n}\right) x_{n}$ so that

$$
\left\|\left(\lambda_{0}-T\right) x_{n}\right\| \rightarrow 0 \text {. }
$$

We now use a lemma due to Heuser, [5] p. 49; if $\alpha\left(\lambda_{0}\right)=\delta\left(\lambda_{0}\right)=p<\infty$, then for all $\lambda \neq \lambda_{0}$ and all integers $n \geqq 0, N\left[(\lambda-T)^{n}\right] \cong R\left[\left(\lambda_{0}-T\right)^{p}\right]$. This enables us to deduce the fact that each $x_{n}$ belongs to $R\left[\left(\lambda_{0}-T\right)^{p}\right]$. If we denote the latter subspace by $R$, then by $R 3, R$ is closed and can hence be considered as a Banach space. It is well known that $\lambda_{0}-T$ maps $R$ onto itself in a one-to-one manner. Let $T_{1}$ be the operator defined in $B(R)$ by $T_{1} x=T x$ for $x \in R$. Then $\lambda_{0} \in \rho\left(T_{1}\right)$. Since $\left(\lambda_{0}-T_{1}\right) x_{n} \rightarrow 0$, it follows that $x_{n} \rightarrow 0$. But $\left\|x_{n}\right\|=1$. Thus we have the required contradiction.

Lemma 2. If we define $R 2^{\prime}: n(\lambda)$ is finite for all $\lambda \neq 0$, then $\mathscr{R}$ is characterised by $R 1$ and $R 2^{\prime}$.

Proof. We need only prove the inequalities $n(\lambda) \leqq n\left[(\lambda-T)^{\alpha(\lambda)}\right]$ and $n\left[(\lambda-T)^{p}\right] \leqq p n(\lambda)$ for all nonnegative integers $p$. The first of these is obvious and the second will be proved by induction. It is clearly valid for $p=0,1$. Assume its validity for $p=k$. Let

$$
N\left[(\lambda-T)^{k+1}\right]=N\left[(\lambda-T)^{k}\right] \oplus Y .
$$

If we can show $\operatorname{dim} Y \leqq n(\lambda)$, our proof is complete.

Suppose $x_{1}, x_{2}, \cdots, x_{s}$ are linearly independent in $Y$. Since these elements belong to $\left.N[(\lambda-T)]^{k+1}\right]$, we know that $\left\{(\lambda-T)^{k} x_{i}\right\}, i=$ $1,2, \cdots, s$, belongs to $N(\lambda-T)$. Suppose $\sum_{1}^{s} c_{i}(\lambda-T)^{k} x_{i}=0$. Then $(\lambda-T)^{k} \sum_{1}^{s} c_{i} x_{i}=0$ so that

$$
\begin{aligned}
& \sum_{1}^{s} c_{i} x_{i} \in Y \cap N\left[(\lambda-T)^{k}\right] \\
\therefore \quad & \sum_{1}^{s} c_{i} x_{i}=0
\end{aligned}
$$

so that all $c_{i}=0$. Hence $\operatorname{dim} Y \leqq n(\lambda)$.

CoROllary. $\mathscr{R}$ consists of those $T$ in $B(X)$ which have the property that, for each $\lambda \neq 0, \alpha(\lambda), \delta(\lambda), n(\lambda), d(\lambda), \bar{d}(\lambda)$ are all finite. Moreover in such case $\alpha(\lambda)=\delta(\lambda)$ and $n(\lambda)=d(\lambda)=\bar{d}(\lambda)$.

This is a consequence of the above lemmas and (1) and (2).

REMARK. In [5], an operator which has the property that, for 
each $\lambda \neq 0, \alpha(\lambda), \delta(\lambda), n(\lambda)$ and $d(\lambda)$ are finite is called "vollfinit". Thus we have identified the class of such operators with $\mathscr{R}$.

Fredholm Operators. $\quad T$ is an operator of Fredholm type in $B(X)$ if $n(T)$ and $d(T)$ are finite. For any $T$ in $B(X)$, the Fredholm region $\Phi_{T}=\{\lambda: \lambda-T$ is of Fredholm type $\}$. It is well known that $\Phi_{T}$ is always an open set.

THeorem. In a space $X$ of infinite dimension, $\mathscr{R}=\{T \in B(X)$ : $\Phi_{T}$ consists of all nonzero complex numbers\}.

Proof. Let $T \in B(X)$ and $\Phi_{r}=\{\lambda: \lambda \neq 0\}$. From [3], Corollary to Theorem 3.3, we have the following fact: If $G$ is a component of $\Phi_{T}$ such that $G \cap \rho(T) \neq \varnothing$, then each point of $G \cap \sigma(T)$ is isolated in $G$. In our case, taking $G=\Phi_{T^{\prime}}$, we can conclude that the nonzero points of $\sigma(T)$ are isolated. Let $\lambda_{0} \in \sigma(T)$ and $\lambda_{0} \neq 0$. If $\lambda_{0}$ is not an eigenvalue, either $\lambda_{0} \in C_{\sigma}(T)$ or $\lambda_{0} \in R_{\sigma}(T)$. But we have seen that $\lambda_{0} \in C_{\sigma}(T)$ implies that $d\left(\lambda_{0}\right)=\infty$, contrary to assumption. Suppose then that $\lambda_{0} \in R_{\sigma}(T)$. Then by the table, we know $n\left(\lambda_{0}\right)=0$ and $d\left(\lambda_{0}\right)>0$. We will show that a neighborhood of $\lambda_{0}$ lies in $R_{\sigma}(T)$, thus contradicting the fact that $\lambda_{0}$ is isolated in $\sigma(T)$. In [7], $\gamma(T)$ is defined for any operator $T$ as the greatest number $k$ for which $\|T x\| \geqq k d(x, N(T))$ for all $x$. Since $n\left(\lambda_{0}\right)=0$,

$$
\gamma\left(\lambda_{0}-T\right)=\inf _{x \neq 0} \frac{\left\|\left(\lambda_{0}-T\right) x\right\|}{\|x\|} .
$$

From [7, p. 272] we know that $\gamma(T)>0$ if and only if $R(T)$ is closed. Since $d\left(\lambda_{0}\right)<\infty$, we know $R\left(\lambda_{0}-T\right)$ is closed so that $\gamma\left(\lambda_{0}-T\right)>0$. Define $N=\left\{\lambda:\left|\lambda-\lambda_{0}\right|<\gamma\left(\lambda_{0}-T\right)\right\}$. Then by Theorem 1 in [7], if $\lambda \in N$ and $\lambda \neq 0$,

$$
n(\lambda) \leqq n\left(\lambda_{0}\right), d(\lambda) \leqq d\left(\lambda_{0}\right)
$$

and

$$
d(\lambda)-n(\lambda)=d\left(\lambda_{0}\right)-n\left(\lambda_{0}\right),
$$

Hence, for $0 \neq \lambda \in N, n(\lambda)=0$ and $d(\lambda)=d\left(\lambda_{0}\right)$. Moreover $\bar{d}(\lambda)=$ $d(\lambda)$ since $d(\lambda)<\infty$, so that

$$
\{\lambda: \lambda \in N, \lambda \neq 0\} \subseteq R_{\sigma}(T) .
$$

Then $\lambda_{0} \in P_{\sigma}(T)$.

Now suppose $E_{0}$ is the spectral projection associated with the spectral set $\left\{\lambda_{0}\right\}$ according to the usual operational calculus described, for instance, in [13]. Then if $T_{1}$ is the operator defined in $B\left(R\left(E_{0}\right)\right)$ 
by $T_{1} x=T x$ for $x \in R\left(E_{0}\right)$, it is well known that $\sigma\left(T_{1}\right)=\left\{\lambda_{0}\right\}$. Moreover, it is obvious that $n\left(\lambda_{0}-T_{1}\right)<\infty$. We will show that $d\left(\lambda_{0}-T_{1}\right)<\infty$. From the decomposition $X=R\left(E_{0}\right) \oplus N\left(E_{0}\right)$, we obtain

$$
\begin{aligned}
R\left(\lambda_{0}-T\right) & =\left[\left(\lambda_{0}-T\right) R\left(E_{0}\right)\right] \oplus\left[\left(\lambda_{0}-T\right) N\left(E_{0}\right)\right] \\
& =R\left(\lambda_{0}-T_{1}\right) \oplus N\left(E_{0}\right)
\end{aligned}
$$

since $\lambda_{0}-T$ maps $N\left(E_{0}\right)$ onto itself. Suppose

$$
R\left(E_{0}\right)=R\left(\lambda_{0}-T_{1}\right) \oplus Y .
$$

Then

$$
\begin{aligned}
X & =N\left(E_{0}\right) \oplus R\left(\lambda_{0}-T_{1}\right) \oplus Y \\
& =R\left(\lambda_{0}-T\right) \oplus Y .
\end{aligned}
$$

Since $d\left(\lambda_{0}-T\right)<\infty, \operatorname{dim} Y<\infty$ and hence $d\left(\lambda_{0}-T_{1}\right)<\infty$. Thus $\Phi_{T_{1}}$ consists of the entire complex plane. According to [3], Theorem 3.2 , this means that $R\left(E_{0}\right)$ is finite dimensional. It is well known that this ensures that $\lambda_{0}$ is a pole of the resolvent $R_{\lambda}(T)$. But this in turn implies that $\alpha\left(\lambda_{0}\right)$ and $\delta\left(\lambda_{0}\right)$ are finite. Hence $R 1$ and $R 2^{\prime}$ satisfied so that $T \in \mathscr{R}$.

On the other hand, if $T \in \mathscr{R}$, then we have by the Corollary to Lemma 2 , that $\Phi_{r}$ contains all nonzero $\lambda$. Since $X$ is infinite dimensional, $\lambda=0$ must be excluded from $\Phi_{t^{\prime}}$ by [3], Theorem 3.2.

REMARK. In a space of infinite dimension, the Fredholm operators form a class disjoint from $\mathscr{R}$. For, if a Fredholm operator belonged to $\mathscr{R}$, its Fredholm region would be the entire plane.

Meromorphic Operators. In $[2,14,15]$ a class $\mathscr{M}$ of operators of so-called meromorphic type are defined by the requirement: $0 \neq \lambda_{0} \in \sigma(T)$ implies $\lambda_{0}$ is a pole of $R_{\lambda}(T)$. It is well known that an isolated point in $\sigma(T)$ is a pole of $R_{\lambda}(T)$ if and only if (i) $\alpha\left(\lambda_{0}\right)$ and $\delta\left(\lambda_{0}\right)$ are finite, and (ii) $R\left[\left(\lambda_{0}-T\right)^{p}\right]$ is closed where $p$ is the common value of $\alpha\left(\lambda_{0}\right)$ and $\delta\left(\lambda_{0}\right)$. From this, it is clear that $\mathscr{R} \subseteq \mathscr{M}$. Moreover $\mathscr{C}$ is characterised by $R 1$ and $R 3^{\prime}$.

$R 3^{\prime}: R\left[(\lambda-T)^{\delta(\lambda)}\right]$ is closed for all $\lambda \neq 0$.

Strictly Singular Operators. The concept of a strictly singular operator was introduced by Kato [7] as follows: a linear transformation $T: X \rightarrow X^{\prime}$ is called strictly singular if, given any subspace $M$ of $X$ such that $T: M \rightarrow T M$ is a linear homeomorphism, then it follows that $M$ is finite dimensional. It is not difficult to see that every compact operator is strictly singular. Examples of strictly singular operators which are not compact were given by Goldberg and Thorp [4]. 
THEOREM. Every strictly singular operator in $B(X)$ is also a Riesz operator.

Proof. Let $T$ be strictly singular in $B(X)$. If $X$ is finite dimensional, the theorem is obviously true. If $X$ is of infinite dimension, we shall show that $\Phi_{T^{\prime}}=\{\lambda: \lambda \neq 0\}$. In [7, p. 285], the idea of one linear operator being strictly singular relative to another is introduced. In our case, it is trivial to verify that $T$ is strictly singular relative to $I$. We now apply Theorem 8 of [7] to obtain: $n(I-\lambda T)$ and $d(I-\lambda T)$ are constant for all $\lambda$ except for a set $S$ which is at most a countable set of isolated points. Moroever, if $n$ and $d$ are these constant values, then $n(I-\lambda T)=n+r(\lambda)$ and $d(I-\lambda T)=d+r(\lambda)$ for each $\lambda$ in $S$, where $r(\lambda)$ is an integer valued function. In particular, since $T \in B(X), n(I-\lambda T)$ and $d(I-\lambda T)$ have constant value zero when $|\lambda| \cdot|| T \|<1$. Thus $n=d=0$. This shows that $n(I-\lambda T)$ and $d(I-\lambda T)$ are finite for all $\lambda$, so that $n(\lambda)$ and $d(\lambda)$ are finite for all nonzero $\lambda$. Thus $\Phi_{r} \supseteqq\{\lambda: \lambda \neq 0\}$ and since $X$ is infinite dimensional, we must have equality. Thus $T \in \mathscr{R}$.

Inessential Operators. In [8], Kleinecke introduced the following notions: let $\mathscr{F}$ represent the closed ideal generated by the set of finite operators in $B(X)$ and write $\phi$ for the canonical homomorphism $B(X) \rightarrow B(X) / \mathscr{F}$. Then $T$ is inessential if $\phi(T)$ lies in the (Jacobson) radical of $B(X) / \mathscr{F}$. The notation used is that of [6] where the quasiradical $q$ and the radical $p$ of an algebra are defined. In the case of an algebra $\mathfrak{A}$ with identity, it is well known that $q$ coincides with the class $\left\{x \in \mathfrak{A}:\left\|x^{n}\right\|^{1 / n} \rightarrow 0\right\}$, i.e., the class of elements whose spectrum consists of the point $\lambda=0$. For a point $\lambda_{0}$ isolated in $\sigma(T)$, Kleinecke gives the following definition: $\lambda_{0}$ has finite multiplicity if there exists a finite projection $E$ such that $T E=E T$ and if $T_{1}$ is the restriction of $T$ to $R(I-E)$, then $\lambda_{0} \notin \sigma\left(T_{1}\right)$. Finally, $T$ has Riesz spectrum if every nonzero point of $\sigma(T)$ is an isolated eigenvalue of finite multiplicity.

LEMma. The class $\mathscr{R}_{\sigma}$ of operators with Riesz spectrum coincides with $\mathscr{R}$.

Proof. Clearly $\mathscr{R}_{\sigma} \supseteqq \mathscr{R}$; for if $T \in \mathscr{R}$, the spectral projections have the required properties so that $T \in \mathscr{R}_{\sigma}$. Conversely, let $T \in \mathscr{R}_{\sigma}$, $0 \neq \lambda_{0}$ be isolated in $\sigma(T)$ and $E_{0}$ be the corresponding projection. It is sufficient to show $n\left(\lambda_{0}\right)$ and $d\left(\lambda_{0}\right)$ are finite. Writing

$$
X=R\left(E_{0}\right) \oplus R\left(I-E_{0}\right),
$$

if $x \in X$, we can write $x=x_{1}+x_{2}, x_{1} \in R\left(E_{0}\right), x_{2} \in R\left(I-E_{0}\right)$. Then, if 
$T x=\lambda_{0} x, T x_{1}+T x_{2}=\lambda_{0} x_{1}+\lambda_{0} x_{2}$ so that $T x_{i}=\lambda_{0} x_{i}, i=1,2$. But $T-\lambda_{0}$ is injective on $R\left(I-E_{0}\right)$. Hence $x_{2}=0$. Thus $N\left(T-\lambda_{0}\right) \subseteq$ $R\left(E_{0}\right)$. Thus $n\left(\lambda_{0}\right)<\infty$. Similarly

$$
\begin{aligned}
\left(\lambda_{0}-T\right) X & =\left(\lambda_{0}-T\right) R\left(E_{0}\right) \oplus\left(\lambda_{0}-T\right) R\left(I-E_{0}\right) \\
& =\left(\lambda_{0}-T\right) R\left(E_{0}\right) \oplus R\left(I-E_{0}\right),
\end{aligned}
$$

Writing $\quad\left(\lambda_{0}-T\right) R\left(E_{0}\right) \oplus Z=R\left(E_{0}\right)$, we have $X=\left(\lambda_{0}-T\right) X \oplus Z$. Since $Z \subseteq R\left(E_{0}\right), d\left(\lambda_{0}\right)<\infty$.

TheoRem. Every inessential operator lies in $\mathscr{R}$.

Proof. This merely restates Thm. 8 of [8].

CoROLlary. The class of inessential operators in a proper subset of $\mathscr{R}$.

For $\mathscr{R}$ is not an ideal, as the examples below make clear.

\section{Perturbation Theory.}

Theorem. (i) If $A, B \in \mathscr{R}$, and $A B=B A$, then $A+B \in \mathscr{R}$. (ii) If $A \in \mathscr{R}$ and $B \in B(X)$ and $A B=B A$, then $A B \in \mathscr{R}$.

(iii) If $\left\{A_{n}\right\}$ is a sequence in $\mathscr{R}$ and $A_{n} \rightarrow A$ in $B(X)$, where $A_{n} A=A A_{n}$ for all sufficiently large $n$, then $A \in \mathscr{R}$.

Proof. In [12], Ruston showed that if $\lambda(T)=\inf \|T-C\|$ where the infimum is taken over all compact $C$, then

$$
\mathscr{R}=\left\{T \in B(X):\left[\lambda\left(T^{n}\right)\right]^{1 / n} \rightarrow 0\right\} \text {. }
$$

If we take $\phi$ to denote the canonical homomorphism $B(X) \rightarrow B(X) / \mathscr{C}$ where $\mathscr{C}$ is the ideal of compact operators in $B(X)$, then we can rewrite the above characterisation as $\mathscr{R}=\{T \in B(X): \sigma[\phi(T)]=\{0\}$. The assertions (i) and (ii) then follow from the known facts:

$$
\begin{gathered}
\sigma[\phi(A+B)] \leqq \sigma[\phi(A)]+\sigma[\phi(B)] \\
\sigma[\phi(A B)] \leqq \sigma[\phi(A)] \cdot \sigma[\phi(B)]
\end{gathered}
$$

when $A B=B A$. For part (iii), we need the fact proven by Newburgh [9]: in any Banach algebra $\mathfrak{A}$, if $\left\{x_{n}\right\}$ is a sequence of elements such that $x_{n} \rightarrow x$ in $\mathfrak{A}$ and $x_{n} x=x x_{n}$ for sufficiently large $n$, then $\sigma\left(x_{n}\right) \rightarrow \sigma(x)$ with convergence in the Hausdorff metric in the plane. In our case, $\phi$ is continuous and hence $\phi\left(A_{n}\right) \rightarrow \phi(A)$ so that $\rho[\phi(A)]=$ $\{0\}$.

REMARK. If we omit the condition $A B=B A$ from (i) and (ii), 
the conclusions are not valid. For suppose $X=l^{\prime}$ and $A$ has matrix representation $\left(a_{i j}\right)$ where

$$
\begin{aligned}
a_{i j} & =\frac{1}{i} \text { when } i=j \\
& =1 \text { when } i=j+1 \text { and } j \text { is even } \\
& =0 \text { elsewhere }
\end{aligned}
$$

and $B$ has matrix representation $\left(b_{i j}\right)$ where

$$
\begin{aligned}
b_{i j} & =-\frac{1}{i} \text { when } i=j \\
& =1 \text { when } i=j+1 \text { and } j \text { is odd } \\
& =0 \text { elsewhere. }
\end{aligned}
$$

Then both $A$ and $B$ belong to $\mathscr{R}$; this can be deduced from [2, pp. 108-111]. Moreover, clearly $A B \neq B A$; for in the matrix representation of $A B$, the $(2,1)$ element equals $1 / 2$, while the corresponding element of $B A$ equals 1 . Now $A+B$ is the operator whose matrix elements are all zero except when $i=j+1$ in which case the matrix element has value 1 . But this operator is considered in $[13, p .266]$ in which it is shown that $\sigma(A+B)$ consists of the entire unit disc $\{\lambda:|\lambda| \leqq 1\}$.

If we now replace $B$ by the operator $B_{1}$ whose matrix representation is $\left(b_{i j}^{\prime}\right)$ where

$$
\begin{aligned}
b_{i j}^{\prime} & =1 \text { when } i=j \text { and } i \text { is even } \\
& =\frac{i-1}{i} \text { when } i=j-1 \text { and } i \text { is even } \\
& =0 \text { elsewhere }
\end{aligned}
$$

we again easily verify that $A B_{1} \neq B_{1} A$ since the $(2,2)$ elements in the matrix multiplications are respectively $1 / 2$ and 1 . Now $I-B_{1} A$ has infinite nullity so that $B_{1} A$ cannot belong to $\mathscr{R}$.

THEOREM. Let $T \in \mathscr{R}$ and $f(\lambda)$ be a function whose domain of holomorphy contains a neighbourhood of $\sigma(T)$. Then if we define $f(T)$ according to the usual operational calculus

$$
f(T)=\frac{1}{2 \pi^{i}} \int_{+B(D)} f(\lambda) R_{\lambda}(T) d \lambda, \quad \text { (see [13, pp. 287-290]) }
$$

then $f(T) \in \mathscr{R}$, whenever $f(0)=0$.

Proof. The present author has shown elsewhere [1] that if $\mathscr{l l}$ is the class of meromorphic operators in $B(X)$ and $f(\lambda)$ is defined as 
above, then $f(T) \in \mathscr{M}$ whenever $T \in \mathscr{L}$. Since $\mathscr{R} \subseteq \mathscr{M}$, we can conclude that $f(T) \in \mathcal{L}$. Hence $R 1$ holds for $f(T)$. If we can prove that $f(T)$ satisfies $R 2^{\prime}$, our proof is complete. Clearly we need show only that if $\mu_{0}$ is a nonzero eigenvalue of $f(T)$, then $n\left(\mu_{0}-f(T)\right)<\infty$. This is equivalent to showing that $R\left(E_{0}\right)$ is finite dimensional, where $E_{0}$ is the spectral projection associated with $\mu_{0}$ and $f(T)$. For if $\alpha\left(\mu_{0}-f(T)\right)=\delta\left(\mu_{0}-f(T)\right)=p$, then it is well known that $R\left(E_{0}\right)=$ $N\left[\left(\mu_{0}-f(T)\right)^{p}\right]$. But we have previously proven that

$$
n\left(\mu_{0}-f(T)\right) \leqq n\left[\left(\mu_{0}-f(T)\right)^{p}\right] \leqq p n\left(\mu_{0}-f(T)\right) .
$$

Hence to complete the proof of this theorem, we need only show that $R\left(E_{0}\right)$ is finite dimensional.

Suppose we write $\left\{\lambda_{k}\right\} k=1,2, \cdots$ for the nonzero points of $\sigma(T)$ and $E_{k}$ for the spectral projection corresponding to $\lambda_{k}$ and $T$. Then since $n\left(\lambda_{k}-T\right)$ is finite for each $k, E_{k}$ has finite dimensional range. But it is possible to deduce from the spectral mapping theorem and the operational calculus that $E_{0}=\sum_{k \in \mathscr{S}} E_{k}$ where $\mathscr{S}=\left\{k: f\left(\lambda_{k}\right)=\mu_{0}\right\}$. See for example Theorem 5.71-C in [13]. Since $\sigma(T)$ has no nonzero points of accumulation, it is easy to see that $\mathscr{S}$ must be a finite set and thus $E_{0}$ has finite dimensional range.

THEOREM. Let $T \in B(X)$ and $f(\lambda)$ be analytic on a region which contains $\sigma(T)$. Suppose that whenever $\lambda_{0}$ is a nonzero element of $\sigma(T), f\left(\lambda_{0}\right) \neq 0$ and that $f(T) \in \mathscr{R}$. Then $T \in \mathscr{R}$.

Proof. If $\lambda_{0}$ is a nonzero element of $\sigma(T)$, then $f\left(\lambda_{0}\right)$ is isolated in $\sigma(f(T))$. Write $\sigma=\left\{\lambda: \lambda \in \sigma(T) ; f(\lambda)=f\left(\lambda_{0}\right)\right\}$. Then it is known (see [13, p. 304]) that $\sigma$ is a spectral set for $T$ and that $E_{\sigma}=E_{0}$ where $E_{\sigma}$ is the spectral projection associated with $\sigma$ and $T$, and $E_{0}$ that associated with $f\left(\lambda_{0}\right)$ and $f(T)$. We know that $f(T) \in \mathscr{R}$ so that $R\left(E_{0}\right)$ is finite dimensional. We can thus consider $R\left(E_{\sigma}\right)$ as a (finite dimensional) Banach space. It is well known that if $T_{1}$ is the restriction of $T$ to $R\left(E_{\sigma}\right)$, then $\sigma\left(T_{1}\right)=\sigma$. Since $R\left(E_{0}\right)$ is finite dimensional, $\sigma$ is a finite set. Since $\lambda_{0} \in \sigma$, this means that $\lambda_{0}$ is isolated in $\sigma(T)$. Moreover, if $E\left(\lambda_{0} ; T\right)$ is the projection associated with $\lambda_{0}$ and $T$ and $E\left(\sigma_{0} ; T\right)$ that associated with $\sigma-\left\{\lambda_{0}\right\}$ and $T$, then

$$
E_{\sigma}=E\left(\sigma_{0} ; T\right)+E\left(\lambda_{0} ; T\right)
$$

with $E\left(\sigma_{0} ; T\right) \perp E\left(\lambda_{0} ; T\right)$. Hence $R\left[E\left(\lambda_{0} ; T\right)\right] \subseteq R\left(E_{\sigma}\right)$ so that $E\left(\lambda_{0} ; T\right)$ has finite dimensional range. When $T$ is restricted to $R\left[E\left(\lambda_{0} ; T\right)\right]$, its resolvent must therefore have a pole at $\lambda_{0}$. But it is known (see [13, p. 313]) that this implies that $\lambda_{0}$ is a pole of $R_{\lambda}(T)$. Finally, since 
$N\left(\lambda_{0}-T\right) \subseteq R\left[E\left(\lambda_{0} ; T\right)\right], n\left(\lambda_{0}-T\right)$ is finite. We can therefore conclude that $T \in \mathscr{R}$.

Theorem. Let $T \in \mathscr{R}$ and $\left\{p_{n}(\lambda)\right\}$ be a sequence of polynomials such that

(i) $p_{n}(0)=0$ for each $n$

(ii) $p_{n}(T)$ converges uniformly to $S$ in $B(X)$. Then $S \in \mathscr{R}$.

Proof. The present author has shown elsewhere [1] that the above theorem holds with $\mathscr{R}$ replaced by $\mathscr{A}$. In our case, this enables us to make the same conclusion and it remains only to prove that $S$ satisfies $R 2^{\prime}$. As previously, this is equivalent to showing that if $\mu_{0}$ is a nonzero point of $\sigma(S)$ and $E_{0}$ is the spectral projection associated with $\mu_{0}$ and $S$, then $R\left(E_{0}\right)$ is finite dimensional. In the proof referred to above, an expression for $E_{0}$ is obtained showing that it is a finite sum of finite dimensional projections. This completes the proof.

We conclude with a theorem similar to those proved by Nikolskii [10].

Theorem. Let $T \in B(X)$ and $R \in \mathscr{R}$ and $T R=R T$. Suppose $0 \in \rho(T+R)$. Then there exists a finite operator $F$ such that $0 \in \rho(T+F)$.

Proof. We can write

$$
T=\left[I-R(T+R)^{-1}\right](T+R)=(T+R)\left[I-(T+R)^{-1} R\right]
$$

since $(T+R)^{-1}$ exists in $B(X)$. Since $R$ commutes with $(T+R)^{-1}$, $(T+R)^{-1} R$ lies in $\mathscr{R}$.

Thus $n\left(I-(T+R)^{-1} R\right)=d\left(I-(T+R)^{-1} R\right)<\infty$.

It is simple to deduce that $n(T)=d(T)<\infty$. The remainder of the proof follows as for Theorem 7 in [8].

\section{BIBLIOGRAPHY}

1. S. R. Caradus, On meromorphic operators (to be published).

2. J. Derr and A. E. Taylor, Operators of meromorphic type with multiple poles of the resolvent, Pacific J. Math. 12 (1962), 85-111.

3. I. C. Gohberg and M. G. Krein, The basic propositions on defect numbers, root numbers and indices of linear operators, Uspehi Mat. Nauk (N.S.) 12 (1957), No. 2 (74), 43118; Amer. Math. Soc. Translations (2) 13.

4. S. Goldberg and E. Thorp, On some open questions concerning strictly singular operators, Proc. Amer. Math. Soc. 14, (1963), 334-336.

5. H. Heuser, Über Operaten mit endlichen Defekten, Inaug. Diss., Tübingen, 1956. 
6. E. Hille and R. S. Phillips, Functional analysis and semigroups, Amer. Math. Soc. Colloq. Publ., Vol. 31, rev. ed. (1957).

7. T. Kato, Perturbation theory for nullity, deficiency and other quantities of linear operators, Journ. d'Anal. Math. VI (1958), 261-322.

8. D. Kleinecke, Almost-finite, compact and inessential operators, Proc. Amer. Math. Soc. 14 (1963), 863-868.

9. J. D. Newburgh, The variation of spectra, Duke Math. J. 18 (1951), 165-176.

10. S. M. Nikolskii, Linear equations in normed linear spaces, Izv. Akad. Nauk SSSR Ser. Mat 7 (1943), 147-166 (Russian).

11. F. Riesz, Über lineare Funktional gleichungen, Acta Math. 41 (1918), 71-98.

12. A. F. Ruston, Operators with Fredholm theory, J. Lond. Math. Soc. 29 (1954), 318-326.

13. A. E. Taylor, Introduction to Functional Analysis, John Wiley and Sons, 1958.

14. - Mittag-Leffler expansions and spectral theory, Pacific J. Math. 10 (1960), $1049-1066$.

15. - Spectral theory and Mittag-Leffler type expansions of the resolvent, Proc. Int. Symp. Linear Spaces, Jerusalem, 1960, 426-440.

Received February 26, 1965. Most of this paper is contained in the author's $\mathrm{Ph}$. D. dissertation, University of California, Los Angeles, 1965. This research was partly supported by the National Science Foundation under Contract GP-1846. 



\section{PACIFIC JOURNAL OF MATHEMATICS}

H. SAMELSON

Stanford University Stanford, California

R. M. BLUMENTHAL

University of Washington

Seattle, Washington 98105

\section{EDITORS}

\author{
*J. DugundJI \\ University of Southern California \\ Los Angeles, California 90007 \\ RICHARD ARENS \\ University of California \\ Los Angeles, California 90024
}

\section{ASSOCIATE EDITORS}

E. F. BeCKENBACH

B. H. NEUMANN

F. WOLF

K. YosidA

\section{SUPPORTING INSTITUTIONS}

UNIVERSITY OF BRITISH COLUMBIA

CALIFORNIA INSTITUTE OF TECHNOLOGY

UNIVERSITY OF CALIFORNIA

MONTANA STATE UNIVERSITY

UNIVERSITY OF NEVADA

NEW MEXICO STATE UNIVERSITY

OREGON STATE UNIVERSITY

UNIVERSITY OF OREGON

OSAKA UNIVERSITY

UNIVERSITY OF SOUTHERN CALIFORNIA
STANFORD UNIVERSITY

UNIVERSITY OF TOKYO

UNIVERSITY OF UTAH

WASHINGTON STATE UNIVERSITY

UNIVERSITY OF WASHINGTON

AMERICAN MATHEMATICAL SOCIETY CHEVRON RESEARCH CORPORATION TRW SYSTEMS

NAVAL ORDNANCE TEST STATION 


\section{Pacific Journal of Mathematics}

Vol. 18, No. 1

March, 1966

Edward Joseph Barbeau, Semi-algebras that are lower semi-lattices ...... 1

Steven Fredrick Bauman, The Klein group as an automorphism group

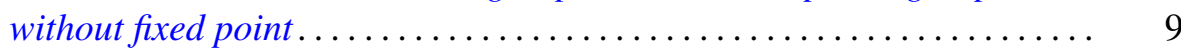

Homer Franklin Bechtell, Jr., Frattini subgroups and $\Phi$-central groups .... 15

Edward Kenneth Blum, A convergent gradient procedure in prehilbert

spaces ............................................

Edward Martin Bolger, The sum of two independent exponential-type random variables ...................................

David Wilson Bressler and A. P. Morse, Images of measurable sets .......

Dennison Robert Brown and J. G. LaTorre, A characterization of uniquely

divisible commutative semigroups........................

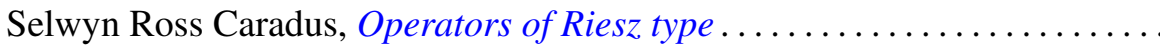

Jeffrey Davis and Isidore Isaac Hirschman, Jr., Toeplitz forms and ultraspherical polynomials ............................

Lorraine L. Foster, On the characteristic roots of the product of certain rational integral matrices of order two ......................

Alfred Gray and S. M. Shah, Asymptotic values of a holomorphic function

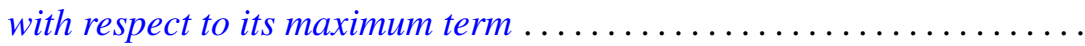

Sidney (Denny) L. Gulick, Commutativity and ideals in the biduals of

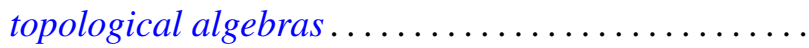

G. J. Kurowski, Further results in the theory of monodiffric functions

Lawrence S. Levy, Commutative rings whose homomorphic images are self-injective .

Calvin T. Long, On real numbers having normality of order $k$....

Bertram Mond, An inequality for operators in a Hilbert space. ...

John William Neuberger, The lack of self-adjointness in three-point boundary value problems ........................

C. A. Persinger, Subsets of $n$-books in $E^{3}$

Oscar S. Rothaus and John Griggs Thompson, A combinatorial problem in the symmetric group ............................... 175

Rodolfo DeSapio, Unknotting spheres via Smale .................. 179

James E. Shockley, On the functional equation

$$
F(m n) F((m, n))=F(m) F(n) f((m, n)) \ldots \ldots \ldots
$$

Kenneth Edward Whipple, Cauchy sequences in Moore spaces ... 